\title{
Dégâts linguistiques collatéraux du français à Istanbul
}

\section{Raymond Renard}

\section{(2) OpenEdition \\ Journals}

Édition électronique

URL : https://journals.openedition.org/dhfles/362

DOI : $10.4000 /$ dhfles.362

ISSN : 2221-4038

Éditeur

Société Internationale pour l'Histoire du Français Langue Étrangère ou Seconde

Édition imprimée

Date de publication : 1 janvier 2007

Pagination : 287-298

ISSN : $0992-7654$

Référence électronique

Raymond Renard, « Dégâts linguistiques collatéraux du français à Istanbul », Documents pour l'histoire du français langue étrangère ou seconde [En ligne], 38/39 | 2007, mis en ligne le 14 décembre 2010, consulté le 27 mai 2021. URL : http://journals.openedition.org/dhfles/362 ; DOI : https://doi.org/ $10.4000 /$ dhfles.362

Ce document a été généré automatiquement le 27 mai 2021.

(c) SIHFLES 


\title{
Dégâts linguistiques collatéraux du français à Istanbul
}

\author{
Raymond Renard
}

\section{Introduction}

1 Mon propos vise à montrer comment la diffusion du français, sous l'égide de l'Alliance israélite universelle (AIU), a contribué au déclin du judéo-espagnol. Je me centrerai essentiellement sur la langue des juifs séphardites du Levant.

\section{Rappel historique}

2 On se rappellera que la prise de Grenade en 1492 par les rois catholiques fut le signal d'un exode de quelques centaines de milliers de membres de la communauté juive d'Espagne. Beaucoup - la moitié, selon Menéndez y Pelayo - passèrent au Portugal, d'où ils allaient d'ailleurs très vite être chassés, faute de se convertir; d'autres opteront pour l'Afrique du Nord ou encore pour des villes d'Europe continentale comme Bordeaux, Amsterdam ou Hambourg. En Italie, où ils furent assez nombreux, une vague de persécutions les chassera au XVI ${ }^{\mathrm{e}}$ siècle. Mais en fin de compte, la destination privilégiée sera l'Empire ottoman, où 70 à 90000 d'entre eux s'installeront entre 1492 et 1600. L'Orient, de tout temps, avait fasciné les juifs, et les Turcs faisaient preuve d'une grande tolérance à leur égard. Déjà au XIVe siècle, suite à des persécutions, nombreux s'étaient réfugiés dans l'Empire byzantin.

Observons que le prince n'a aucun intérêt à convertir les juifs car la tolérance dont il fait preuve est assortie du payement d'un impôt spécial : la djizya! (Dans le monde de l'époque, la règle était celle du cujus regio ejus religio, que Louis XIV traduira par la formule « un roi, une foi, une loi »). Lors de la prise de Byzance en 1453, les Ottomans n'avaient-ils pas épargné le quartier juif de Balat?

4 Au XVIe siècle, il y avait 44 cales (synagogues) à Constantinople (dont une lisboeta et une barcelonesa). D'emblée, l'activité débordante des séphardim, leur ardeur au travail, 
jointes à leurs vastes connaissances, leur assurèrent une grande prospérité, ainsi qu'un incontestable prestige auprès d'autres communautés ethniques. De nombreux séphardim occupèrent vite d'importantes situations dans l'Empire, qu'ils servirent fidèlement. Leur sort fut particulièrement enviable sous les sultans Bajazet II, Sélim $1^{\mathrm{er}}$ et Soliman II. Ils constituent alors la bourgeoisie et la classe marchande de la société ottomane. Ils détiennent le commerce de gros: les négociants juifs d'Istanbul, Salonique ou Smyrne ont des agents dans le monde entier, même en Espagne... Comme ils contrôlent en outre l'artisanat, le commerce de détail est pratiquement entre leurs mains.

5 Sur le plan culturel, le rayonnement des séphardim n'est pas moins grand : ils fondent les premières imprimeries en Orient, en 1506 à Istanbul. L'université de Salonique, créée par eux, voit affluer vers elle des savants et des étudiants du monde juif tout entier. On vient y étudier le droit, l'astronomie, la philosophie, le Talmud... Les séminaires de Salonique forment des rabbins pour les communautés séphardites de toutes les régions.

6 La culture judéo-espagnole d'Orient sera loin d'être négligeable (cf. Molho, 1960). Le domaine de la littérature orale est particulièrement digne d'intérêt, avec ses nombreux refranes et son romancero: les romances judéo-espagnoles, "véritable prodige de traditionnalisme» (Menendez Pidal, 1958), qui remontent au moyen âge et aussi, plus récents, les romances, d'inspiration biblique ou encore les cantares amorosos nés vers la fin du XIX siècle.

7 Dans le domaine de la littérature écrite, les exilés n'ont jamais retrouvé le haut niveau culturel atteint dès l'époque d'Alphonse X. Limitons-nous à signaler, au XVI ${ }^{\mathrm{e}}$ siècle, le chef-d'œuvre du Saloniquais Almosnino, Regimiento de la Vida, au XVIII', le Meam Lo'ez, encyclopédie populaire qui exerça longtemps une grande influence sur les masses.

8 La prospérité dans l'Empire dura jusqu'à la fin du XVI eiècle. La décadence devait survenir rapidement, suite à la conjonction de différents facteurs: perte de l'importance des grands ports de la Méditerranée après les grandes découvertes, appauvrissement de la vie intellectuelle, faute de contacts avec l'Occident alors en pleine Renaissance.

9 En réalité, la vie intellectuelle souffrait de l'absence d'un système éducatif suffisamment efficace. L'enseignement se limite aux meldares rabbiniques, où l'on apprend aux seuls garçons à lire quelques prières et des fragments de la Bible. On leur apprend aussi à écrire, mais comme le papier est cher, c'est sur le sable qu'ils s'exerceront.

10 Dès la fin du XVIII ${ }^{e}$ siècle, rares étaient les juifs qui occupaient une fonction dans l'administration, aussi les sultans se montraient-ils de moins en moins enclins à les protéger, par exemple, contre les exactions des janissaires, particulièrement cruels envers les juifs, surtout à Constantinople ${ }^{1}$.

11 La fin du XIX et le $\mathrm{XX}^{\mathrm{e}}$ siècles marqueront l'ultime dislocation du monde judéoespagnol, qui ne résistera pas à l'effondrement de l'Empire ottoman et à la poussée nationaliste qui en résulta. Dans les nouveaux États, l'intolérance religieuse reprit une vigueur nouvelle qui entraîna, en provenance de Macédoine, de Grèce ou de Bulgarie, un renforcement de l'élément juif de Turquie, Istanbul surtout (où ils occupaient le quartier de Galata). Ataturk leur donna un statut, mais prit des mesures de turkisation. Le premier recensement de la République, en 1927, en dénombre 80000 . Leur 
population croît encore ensuite pour tomber à 25000 après la Deuxième Guerre mondiale (Shaw, 1992).

\section{Aspect géolinguistique} demeurés fidèles, des siècles durant, au langage de leurs ancêtres. Les premiers temps de l'exil - le castillan avait alors accompli l'essentiel de son évolution phonétique ${ }^{2}$, - il ne cessa cependant de se transformer, suivant le cours de sa propre évolution. massif -, s'était élaborée une cursive sans doute moins belle, mais beaucoup plus pratique car plus simple de forme : l'écriture rabbinique, souvent appelée écriture rachi (Foulché-Delbosc 1894 : 23).

17 Ce système avait été adopté par la majorité des communautés du monde judaïque, quelle que fût la langue parlée: arabe, persan, tartare, ancien français, ancien provençal, ancien allemand (devenu yiddish), italien ou espagnol. Pour les juifs, il possédait donc, plus que tout autre, un caractère d'universalité. L'ignorer eût été commettre un véritable sacrilège et rompre, en quelque sorte, avec la Loi. même de leur attachement à la langue castillane (qui au Levant s'était très vite imposée aux autres communautés ibériques). Si ceux d'Occident (Europe occidentale et Italie) adoptèrent l'alphabet latin dès l'époque de la Renaissance, réservant l'alphabet rachi aux usages religieux, ceux d'Orient ne connurent, jusqu'à une époque fort récente, que l'alphabet rabbinique.

19 Les ouvrages qui s'imprimèrent nombreux, surtout au XVI ${ }^{\mathrm{e}}$ siècle, dans l'Empire ottoman, le furent en caractères hébraïques. On enseignait cette écriture dans toutes les écoles juives et c'était un devoir religieux de l'apprendre avant n'importe quelle autre.

Circonstance fut déterminante aux points de vue linguistique et culturel car elle coupa définitivement le contact avec la culture de la mère-patrie, ruina le goût de la composition, limita aussi l'essor littéraire et culturel.

21 Sur le plan linguistique proprement dit, l'utilisation de l'alphabet hébraïque devait engendrer une dégradation supplémentaire de la langue.

Non seulement elle détruisit davantage toute possibilité de puiser au fonds lexicologique de la Péninsule et provoqua des emprunts innombrables à l'hébreu, au 
turc, au français, à l'italien ou aux langues balkaniques, mais elle affecta la prononciation.

L'alphabet hébraïque, en effet, comme d'ailleurs celui de toutes les langues sémitiques, est relativement pauvre. Ses vingt-deux signes représentent uniquement des consonnes, quatre d'entre eux pouvant être utilisés pour exprimer l'allongement des voyelles. Celles-ci, quand elles sont brèves, doivent être suppléées par le lecteur. Un tel système ne peut évidemment reproduire le vocalisme espagnol. D'autant moins que certains de ses signes représentent des sons étrangers à la phonétique castillane ou font, au point de vue de celle-ci, double emploi entre eux.

Ajoutons enfin que, dans les derniers temps, la cursive rachi ne permettait même plus une compréhension parfaite entre toutes les communautés séphardites, tant elle s'était diversifiée de région à région.

On comprendra donc que le système de transcription propre aux séphardim ne pouvait contribuer à préserver la pureté du castillan. Au contraire, on peut imputer dans une large mesure à l'utilisation de l'alphabet rachi la tendance au relâchement et à l'imprécision du système phonique judéo-espagnol.

Lorsqu'au $\mathrm{XX}^{\mathrm{e}}$ siècle, sous l'influence d'Ataturk, les séphardim de Turquie durent abandonner leurs caractères pour l'alphabet latin ${ }^{4}$, c'est l'adaptation turque ${ }^{5}$ qu'on leur imposa et non la castillane! Si ce procédé marque un progrès par rapport au système rachi, il n'en est pas moins mal adapté à la langue parlée.

Il s'en faut de beaucoup que cette confusion, - caractéristique de l'imprimerie judéoespagnole en Turquie, - ait contribué au respect de l'orthographe ou à l'observance des règles les plus élémentaires du métier par les typographes, qui n'en furent pas à une coquille près ${ }^{6}$ !

Lors du lancement du journal El Tiempo, de Tel Aviv, en 1950, les premiers numéros furent imprimés avec l'orthographe espagnole. Les lecteurs les retournèrent si nombreux qu'on utilisa l'orthographe phonétique: sospetchar, dicho (dijo), ajente, dirigente, yamar, aktitud, anio, egzamen...

\section{L'action de l'Alliance israélite universelle (AIU)}

$\mathrm{Au}$ XIX ${ }^{\mathrm{e}}$ siècle, la situation culturelle des juifs espagnols n'était guère brillante, du fait notamment, on l'a vu, de l'inexistence d'un système éducatif crédible.

Dans la seconde moitié du siècle, un puissant mouvement d'émancipation intellectuelle parti d'Occident et accompagné d'un renouveau économique va secouer les communautés juives d'Orient et les sortir de l'état de léthargie. Il s'agit de la décision de l'AIU, fondée à Paris en 1860, de créer des écoles françaises à l'intention des juifs ${ }^{7}$. Dès 1862, des écoles de l'AIU s'établiront à Tétouan (1862), Tanger (1864), Istanbul et Bagdad (1865), Andrinople (1867), Schoumla (1870), Smyrne (1873), Samokov (1874), Plovdiv (1881), Sofia (1887), Monastir (1895).

31 Inspirée par l'«idéal humaniste républicain » et les "principes de 89 », l'AIU veut " apporter un rayon de civilisation de l'Occident dans les milieux dégénérés par des siècles d'oppression et d'ignorance" (Pellandra 2001: 14). Pour cela, le «français, introduit progressivement, est la langue d'enseignement, sans pourtant oublier la langue du pays, l'intégration dans la communauté nationale étant le but principal de 
l'Alliance » (Spaëth 2001 : 108-111, cité par Pellandra, p. 14). En dépit de la résistance des rabbins, ces écoles répondaient à un véritable besoin et elles connaîtront un grand succès ${ }^{8}$. En 1895, l'AIU possède 59 écoles avec 12050 élèves ${ }^{9}$. Ceux-ci seront plus de 50000 en 1953. Mis en contact avec des langues et des civilisations nouvelles, les séphardim se rendent compte de l'immense pauvreté de leur langue. Dès lors, ceux d'entre eux qui ne veulent pas se résoudre à abandonner le judéo-espagnol puisent sans ordre ni mesure dans les langues de culture qu'ils connaissent et l'on va assister à une francisation du judéo-espagnol.

L'invasion des gallicismes sera courante dans la langue parlée aussi bien qu'écrite, bien que la première ait été moins atteinte que la seconde.

L'influence de cet enthousiasme en faveur du français fut telle sur un nombre de plus en plus important de séphardim avides de culture qu'ils se transformèrent en bilingues à prédominance française (cf. Mezan, 1925).

En 1932, il était courant de rencontrer dans les familles judéo-espagnoles des grandsparents hispanophones incapables de converser avec leurs petits-enfants francophones (Galanté $1932: 151)$.

La lecture des périodiques produits au Levant dans la seconde moitié du XXe siècle est particulièrement édifiante: elle donne une idée du relâchement de la langue judéoespagnole.

On en jugera par cet extrait de La Boz de Türkiyé « Revista mensual de informaciones, científica y literaria », paru à Istanbul en 1946 (Anio VII) :

La Tragedia Judia en Europa y la Ayuda de la J.D.C.

Del primo enero hasta noviembre 1945, la Joint Distribution Committe, la grande Sociedad americana de relevamiento y de socorros, apartó 25.626.225 Dollares para los socoros en Europa y otros centros judíos en desdicha. Esto representa algunos 7 millones de más comparativamente al montante apropriado en 1944, que era considerado como la aniada de los más grandes gastes en la historia de la J.D.C.

Pero mismo así, fueron lejos de pueder asigurar abastansa los menesteres presentados a esta sociedad. Solo aquellos que vieron con sus propios ojos y sentieron el golpe de las yagas de cada día de un million 250.000 judíos de Europa que sovrevieron en Europa continental pueden apreciar la urgencia del problema del ayudo americano. Tragicamente los judíos de Europa que sobrevivieron a la terror demandan ahora mantinimiento y vestimientas afin de pueder pasar el hinvierno. Ellos hacen llamada por obtener abrigos para las criaturas, casas en lugar de cavanias, ayuda medical y la chance de lavorar o de embezar a lavorar de nuevo. La J.D.C. hizo todo lo posible con los fondos que ella posedava. Pero con más grandes medio sella uviera podido enviar más grandes cuantidades de ayuda vital, establecar muchos más projetos de reconstrucción y sociedades de prestimos, y enfin ouviera emprendido un programa de rehabilitación más apropiado.

37 À côté des traits caractéristiques du judéo-espagnol, comme les irrégularités de la diphtongaison, on notera de nombreux gallicismes :

- des mots transposés du français et dont l'emploi est injustifié en raison de l'existence de termes castillans ;

- des formes correctes, certes, mais d'usage peu courant en espagnol, et qui ont sans doute été préférées à cause de leur parenté avec le français ;

- des influences orthographiques ;

- l'absence totale de formes syntaxiques si chères à la langue espagnole comme l'inversion du sujet et du verbe et l'intrusion de pronoms personnels sujets. 
D'autres publications de la même époque, nous avons relevé un certain nombre d'emprunts ou d'anomalies, repris dans le tableau ci-après :

\begin{tabular}{|c|c|c|}
\hline Judéo-espagnol & français & castillan \\
\hline Madama, madmazel, musiú & Mme, Mlle, M. & Sra, Srita, Sr. \\
\hline Es ansi ke & C'est ainsi que & Asi es que \\
\hline El mundo & Les gens & La gente \\
\hline malgrado & malgré & A pesar de \\
\hline jurnal & journal & periódico \\
\hline ma & mais (it. ma) & pero \\
\hline de mas en mas & de plus en plus & cada vez más \\
\hline se arresto & il s'arrêta & se paró \\
\hline enregistrado & enregistré & registrado \\
\hline en (kon) el buto de & dans le but de & afin de \\
\hline sus fuyida & sa fuite & su huida (fuga) \\
\hline se pensa & on pense & se piensa \\
\hline frontiera & frontière & frontera \\
\hline esforzo & effort & esfuerzo \\
\hline manifestos & manifestes & manifiestos \\
\hline rempuchar & repousser & rechazar \\
\hline enkuesta & enquête & investigación \\
\hline inyorar & ignorer & ingnorar \\
\hline a $15 \mathrm{~km}$ & à 15 km & a los $15 \mathrm{~km}$ \\
\hline suetar, dezer & désirer & desear \\
\hline enverso esta lengua & envers cette langue & para esta lengua \\
\hline en tala mizura & en telle mesure & con tal medida \\
\hline chomaje, chomores & chômage,chômeurs & paro, parados \\
\hline malhorozo & malheureux & infeliz \\
\hline sentura & ceinture & cinturón, cintura \\
\hline buro & bureau & oficio \\
\hline sjemindefer & chemin de fer & ferrocarril \\
\hline
\end{tabular}

\section{Conclusion}

Voici plus d'un demi-siècle, Max L. Wagner (1950: 15) diagnostiquait le judéo-espagnol comme una lengua moribunda. Il ne se trompait pas, si l'on admet que cette langue, demeurée familiale dans certaines communautés de la diaspora sephardite, n'est pratiquement plus parlée par les jeunes générations, qui ont résolument adopté les langues locales, - turc ou hébreu.

Le français est-il la cause de son dépérissement? 

nous découvrons en premier lieu des facteurs économiques et politiques : la cohésion des séphardim, qui explique en grande partie leur fidélité à leur langue, résista mal à la crise économique que connut la Méditerranée orientale à la suite de l'industrialisation des grandes nations occidentales; elle fut soumise à rude épreuve lors de la chute de l'Empire ottoman, la constitution des nouveaux États balkaniques, la Deuxième Guerre mondiale et la création de l'État d'Israël. Cette cohésion souffrit également de l'affaiblissement du sentiment religieux chez beaucoup d'intellectuels. culturel et linguistique. Les progrès de l'instruction publique dès la fin du XIX ${ }^{\mathrm{e}}$ siècle et la carence des séphardim en cette matière ainsi que le prestige accru de certaines grandes langues internationales - le français d'abord, l'anglais ensuite -, entraînèrent une véritable crise de confiance des séphardim dans l'avenir de leur langue, qu'un lexique de base indigent, un système imprécis de transcription et la carence d'autorité en matière linguistique avaient condamnée à la sclérose et à l'abâtardissement.

On ne peut manquer de souligner l'importance du prestige du français dans l'accélération du processus de désintégration du judéo-espagnol. La création d'écoles par l'Alliance israélite universelle fut déterminante à cet égard.

Mais, en ouvrant ses écoles, l'AIU ne poursuivait certainement pas un objectif linguicide, mais au contraire visait à permettre à des populations culturellement défavorisées l'accès à la modernité.

Malheureusement pour le patrimoine de l'humanité que constitue la diversité linguistique, l'enfer est pavé de bonnes intentions, et les meilleures intentions entraînent souvent d'importants « dégâts collatéraux ».

\section{BIBLIOGRAPHIE}

ALHADEFF (1991). « Les communautés séphardites sous l'Empire ottoman », Los Muestros, 4, mars 1991, 10-11; 5, déc. 1991, pp. 10-11.

Alliance Israélite Universelle (L') (1860-1895). Paris, s. d. [AIU].

ANTOINE, Gérald et MARTIN, Robert (éd.) (1985). Histoire de la langue française (1880-1914), Paris, CNRS.

DUMONT, Pierre (1999). La politique linguistique et culturelle de la France en Turquie, Essai, Paris, L'Harmattan.

FOULCHÉ-DELBOSC, Raymond (1894). « La transcription hispano-hébraïque », Revue hispanique, I, pp. 22-33.

GALANTÉ, Avram (1932). Turcs et Juifs, étude historique, politique, Istanbul, Haim, Rozio et C. KOL-SEPHARAD, revue de la Fédération séphardite mondiale, Londres, 1958-1967. 
LÉVY, Francine (1990). « L'œuvre de l'Alliance Israélite Universelle », Études de Linguistique Appliquée, 78,pp.97-108.

MENENDEZ PIDAL, R. (1958). Los romances de América y otros estudios, Madrid, Espasa-Calpe. MÉZAN, Saül (1925). Les Juifs espagnols en Bulgarie, Sofia, Amischpat.

MOLHO, M. (1960). Literatura Sefardita de Oriente, C.S.I.C., Instituto Arias Montano, Bibl. hebraicoespañola, vol. VII, Madrid, Barcelona.

PELLANDRA, Carla (2001). « La diffusion du français dans le bassin de la Méditerranée de 1880 à 1914 », SIHFLES, Documents pour l'histoire du français langue étrangère ou seconde, 27, 11-32.

PEREZ, Claude (1992). «Éléments pour une histoire des écoles francophones en Turquie », SIHFLES, Documents pour l'histoire du français langue étrangère ou seconde, 10, 43-49.

SEMERCIOĞLU, Ufuk (1992). «Origine de l'enseignement du français en Turquie : le Lycée impérial ottoman », SIHFLES, Documents pour l'histoire du français langue étrangère ou seconde, 10, 50-59.

SHAUL, M. (décembre 1991). «El Djudeo-Espanyol en nuestros días », Los Muestros, 5, 59.

SHAW, St. J. (mars 1992). « The story of Turkish Jewry : five hundred years of Peace and Harmony ", Los Muestros, 6, 17-21.

SIHFLES (2001-2002). Documentspour l'histoire du français langue étrangère ou seconde, Histoire de l'enseignement du français langue étrangère ou seconde dans le bassin méditerranéen, 27, 28.

SPAËTH, Valérie (1985). « La création de l'Alliance israélite universelle hors des pays francophones ou francisants ", in G. ANTOINE et R. MARTIN (éd.), op. cit., pp. 421-432.

WAGNER, M.-L. (1930). Caracteres generales del judeo-español de Oriente, R.F.E., Anejo XII, Madrid.

WAGNER, M.-L. (1950). « Espigueo judeo-español », Revista de Filología Española, XXXIV.

\section{NOTES}

1. Il faut dire que la communauté de Constantinople, très importante numériquement et influente dans le commerce « avait, en plus, le privilège d'envoyer des députés au parlement et des conseillers à la cour et aux ministères " (Alhadeff, 1991, 5). Au début du XX ${ }^{\mathrm{e}}$ siècle, Salonique était une ville presque espagnole, où les juifs se trouvaient plus nombreux que les Grecs, les Turcs et les Bulgares réunis (Molho, 1960, 144).

2. La première grammaire, celle de Nebrija, date de 1492.

3. Sous la domination perse, l'usage de la langue et de l'écriture araméennes s'était étendu à tout l'Orient.

4. Loi du 3 novembre 1928.

5. Les séphardim de Salonique choisirent, eux, l'alphabet latin francisé !

6. Je trouve dans un articulet de 21 lignes annonçant un accident d'aviation et paru dans La Vera Luz du 7 juin 1962 (p. 3) le terme «catastrophe" orthographié de trois manières: katastrofa, katistrofa, katostrafa. C'est par centaines que l'on pourrait dénombrer les fautes d'impression dans les journaux judéo-espagnols contemporains.

7. Le gouvernement italien ouvrira des écoles italiennes. Signalons qu'en 1854 déjà, Albert Cohen, attaché à la maison Rothschild de Vienne, avait créé une première école primaire juive à Piri Pacha. Le turc, l'hébreu et le français y sont enseignés. Les rabbins, furieux, excommunièrent le banquier Camondo qui l'avait prise en charge en 1862 . 
8. La création en 1868 du lycée impérial ottoman (Galatasaray) connaîtra le même succès auprès de l'élite, imprégnée de culture française.

9. Sur ces 59 écoles, 22 de filles avec 4900 élèves (AIU).

\section{RÉSUMÉS}

Sous ce titre, notre exposé tente de montrer comment la diffusion du français dès la fin du XIX siècle dans la société levantine juive a contribué au déclin du judéo-espagnol.

On se rappellera que la prise de Grenade en 1492 par les rois catholiques fut le signal d'un exode de quelques centaines de milliers de membres de la communauté juive d'Espagne. Leur destination privilégiée fut l'Empire ottoman, qui fit preuve d'une grande tolérance à leur égard. De telle sorte que des villes comme Salonique, Istanbul ou Smyrne connurent, grâce aux juifs, une grande prospérité jusqu'à la fin du XVI ${ }^{\mathrm{e}}$ siècle. La langue judéo-espagnole était, en quelque sorte, la langue des affaires du monde ottoman. Mais l'Empire ottoman allait entraîner toutes ses composantes dans une longue décadence. Lorsqu'au XIX ${ }^{\mathrm{e}}$ siècle, l'Alliance israélite universelle créera une chaîne d'écoles dans les principales cités levantines, les séphardim seront nombreux à les fréquenter. Le succès du français auprès des élites juives sera tel qu'il est permis de lui attribuer une bonne part du déclin du judéo-espagnol, ainsi qu'on pourra le constater à l'analyse de périodiques locaux du milieu du $\mathrm{XX}^{\mathrm{e}}$ siècle.

This study is aiming to explain how the spreading of French contributed to the decline of the Judaeo-Spanish language in the Levantine Jewish society as early as the end of the $19^{\text {th }}$ Century. It is to be remembered that the taking of Granada by the Catholic kings was the signal for the exodus of hundreds of thousands of members of the Jewish community out of Spain. The privileged destination was then the Ottoman Empire, which was very accepting to this population. It is the reason why, thanks to the Jews, towns such as Salonique, Istanbul or Smyrne were very prosperous until the end of the $16^{\text {th }}$ Century. At that time, the Judaeo-Spanish was, so to say, the "business language » of the Ottoman society. However, the Ottoman Empire was to take all its components along with it into its long declay. When the Alliance Israélite Universelle created a range of schools in the most important Levantine cities, a lot of Sephardim attended them. The success of French among the Jewish elite was such that it is considered to be mostly responsible of the decline of the Judaeo-Spanish language. The analysis of local publications dating of the middle of the $20^{\text {th }}$ Century supports this assumption.

\section{INDEX}

Mots-clés : déclin du judéo-espagnol, diffusion du français, Empire ottoman, séphardim

Keywords : decline of the Judaeo-Spanish language, Ottoman Empire, Sephardim, Spreading of French language 
AUTEUR

RAYMOND RENARD

Université de Mons-Hainaut, Belgique 